Recherches en histoire de l'art, histoire des civilisations, archéologie, anthropologie et muséologie

$16 \mid 2021$

Cahier $n^{\circ} 16$

\title{
Éditorial
}

Varia, échanges

\section{François-René Martin}

\section{(2) OpenEdition}

12 Journals

Édition électronique

URL : https://journals.openedition.org/cel/16375

DOI : $10.4000 /$ cel. 16375

ISSN : 2262-208X

Éditeur

École du Louvre

\section{Référence électronique}

François-René Martin, «Éditorial », Les Cahiers de l'École du Louvre [En ligne], 16 | 2021, mis en ligne le 28 juillet 2021, consulté le 30 juillet 2021. URL : http://journals.openedition.org/cel/16375 ; DOI : https://doi.org/10.4000/cel.16375

Ce document a été généré automatiquement le 30 juillet 2021.

\section{(c) (i) (9)}

Les Cahiers de l'École du Louvre sont mis à disposition selon les termes de la licence Creative Commons Attribution - Pas d'Utilisation Commerciale - Pas de Modification 4.0 International. 


\title{
Éditorial
}

\author{
Varia, échanges
}

\section{François-René Martin}

1 C'est un nouveau numéro de varia que cette $16^{\mathrm{e}}$ livraison des Cahiers de l'École du Louvre propose. Il rend compte, une fois encore, de la variété des domaines que couvrent lesdoctorants de l'École, principaux pourvoyeurs des travaux édités dans cette publication. Si les études qui ont été ici réunies concernent pour l'essentiel la période contemporaine, de la Révolution française à la fin du $\mathrm{XX}^{\mathrm{e}}$ siècle, ce sont les genres mêmes d'écriture qui expriment le mieux cette variété. À côté des études «traditionnelles» qui viennent faire le point sur une question savante - les femmes artistes ou la situation de la critique en Allemagne après 1945 -, on trouvera un essai venant croiser numismatique et histoire monétaire, ainsi qu'un autre alliant à une étude lexicologique les acquis de la génétique des textes littéraires, transportés sur une question artistique. Enfin, dans des formats augmentés, une longue étude sur un chefd'œuvre délaissé de la sculpture du XIXe siècle, issue d'une conférence donnée à l'École française d'Athènes, reprise à l'École du Louvre, ainsi que le catalogue raisonné du fonds de dessins de presse d'un artiste du même siècle, conservé dans un musée.

2 Conservateur général du patrimoine honoraire, ancien directeur de l'École du Louvre, c'est en spécialiste de la sculpture du XIX siècle que Philippe Durey ( « La Jeune Grecque de David d'Angers ou le rêve brisé ») se penche sur une œuvre mal connue du sculpteur. La genèse de l'œuvre est éclairée dans l'étude : profondément marqué par la mort de Marco Botzaris, le héros de l'indépendance grecque mort au combat lors du second siège de Missolonghi en 1823, David d'Angers en vint à concevoir un monument qui est dans le domaine de la sculpture l'équivalent de La Grèce sur les ruines de Missolonghi (Bordeaux, Musée des Beaux-Arts) ou des Scènes des Massacres de Scio (Paris, Musée du Louvre). Il est sans doute injuste, comme le fait remarquer Ph. Durey, que la sculpture n'ait pas eu la même fortune critique que les peintures philhellènes d'Eugène Delacroix. Histoire de médium, avant tout, et que les travaux menés dans le domaine de la sculpture, depuis bientôt un demi-siècle, ne sont pas encore parvenus à corriger l'intérêt de cette étude n'en est que plus fort. La «biographie » de cette œuvre ensuite, sa trajectoire, ainsi que celle de David d'Angers, l'expliquent. Delacroix l'a emporté. 
Pourtant, cette jeune grecque dont la légende dit que c'est une jeune fille pauvre de Montparnasse, rencontrée par hasard dans la rue, qui donna à David et l'idée du monument et sa physionomie, est en réalité la sœur, plus que des personnages féminins des grandes compositions épiques, de l'autre grand chef-d'œuvre qu'est La Jeune orpheline au cimetière (Paris, Musée du Louvre). David donna donc à la Grèce, à la suite de tractations complexes, cette sculpture dont la datation peut être précisée. C'est en 1837, deux années après son arrivée sur le sol hellène, que le monument La Jeune Grecque fut placée dans le jardin des Héros, à Missolonghi. Elle en fut enlevée en 1915, remplacée par une copie, pour le Musée national historique d'Athènes, où l'on peut la voir aujourd'hui. Au-delà de la reconstitution minutieuse du dossier de l'œuvre et des aléas de son transfert en Grèce, c'est un chapitre du philhellénisme français qui est reconstitué dans cette étude, aboutissant au séjour de David d'Angers en 1852, l'exilé républicain ayant rêvé créer une école d'art dans la patrie de Phidias. Triste voyage de vieillesse, où le sculpteur se pencha sur sa sculpture mutilée et délaissée par les autochtones...

«Une constellation dans la nuit de l'histoire, une tache lumineuse devant les ténèbres de la mort: l'œuvre d'Ernst Wilhelm Nay vue par Werner Haftmann». L'étude de Vincenza Benedettino traite d'un des couples artiste/critique les plus importants de la scène artistique allemande après 1945 . Et à travers ce couple, d'un des moments les plus passionnants et complexes de l'art moderne européen et américain, placé sous le signe d'une abstraction qui apparaissait alors comme le seul langage propre à satisfaire l'injonction très partagée à « repartir à zéro ", pour reprendre l'expression de Barnett Newman. On sait qu'avant que ne se forme l'entente, faite à la fois d'amitié et de stratégie, entre Nay, artiste formé auprès de Karl Hofer et Georg Karl Heise, et le critique Werner Haftmann, un autre duo avait régné sur l'art moderne allemand de l'immédiat après-guerre: Willi Baumeister et Will Grohmann. D'une certaine façon, l'association entre l'historien de l'art et homme de musée, et l'artiste qui explore à ce moment une nouvelle voie vers l'abstraction, vient prendre la relève du premier couple. Lors de la première Documenta, en 1955, organisée par Arnold Bode et à laquelle Haftmann est étroitement associé, l'on salue la mémoire de Baumeister qui vient de disparaître et l'on assiste à la «canonisation» de Nay, qui passe alors au premier plan dans la promotion d'un art abstrait allemand. La publication de Malerei im 20. Jahrhundert, en 1954, la synthèse de grande diffusion du même Haftmann, avait déjà fixé la doctrine de cette modernité de rupture. Par la suite, comme l'explique avec clarté V. Benedettino, Haftmann continuera à défendre et Nay et l'abstraction dans ses ramifications complexes au risque d'en venir à disqualifier les courants nouvellement venus, en Allemagne comme ailleurs, au nom d'une théorie des générations artistiques forcément dépendante de celle d'un Wilhelm Pinder. Derrière la rupture moderniste, l'on devine les vieilles idées et parfois même les adhésions précoces. La jeunesse de Haftmann est aujourd'hui interrogée, avec ses engagements enfouis, loin de l'image qui fut longtemps la sienne. Cela ne rend que plus importante cette étude et les travaux qui vont la prolonger.

4 C'est la question très débattue depuis quelques années des femmes artistes aux XVIII ${ }^{\mathrm{e}}$ et XIX siècles que reprend Vera de Ladoucette, en étudiant pour commencer la présence des femmes dans les salons. Deux tableaux : L'Autoportrait avec deux élèves (New York, Metropolitan Museum), d'Adélaïde Labille-Guiart, exposé au Salon de 1785 et le Tableau représentant feue Madame Vincent, élève de son mari, de Marie-Gabrielle Capet (Munich, Neue Pinakothek), présenté au salon de 1808 et par lequel une des deux élèves 
placées derrière Adélaïde vient à son tour représenter celle qui avait été sa professeure de peinture. Ces deux bornes qui délimitent un quart de siècle ou quasiment permettent de mesurer des évolutions, la part des femmes exposées au Salon étant de 6,3 \% lors du premier Salon de la Révolution pour croître jusque 15,4 \% en 1802. Mais cet indicateur mérite d'être croisé avec un autre, qui le nuance: le nombre des autoportraits proposés au regard du visiteur parmi les tableaux de ces artistes femmes, qui est parfois considérable dès le début de la période. Si on le rapporte au nombre d'autoportraits d'artistes hommes, en 1793, pour ne prendre qu'un exemple, les deux parts sont égales : les femmes sont aussi nombreuses à s'y représenter, s'y afficher dans leur vocation et leur métier, que les hommes. Ces deux variables permettent d'esquisser une analyse de ce moment historique dans ces contradictions: l'augmentation du nombre de femmes artistes - et sans doute la relative démocratisation de l'accès à la profession - irait, selon l'auteure de l'étude, avec un effacement progressif de la revendication de la femme peintre en tant qu'artiste. C'est du reste dans la mise en relation des deux tableaux indiqués en ouverture, où sont mises en scène toutes sortes de dépendances et de dettes, que l'on verrait mieux cette mutation. Là où l'artiste s'affichait fièrement dans son activité, sous son nom de femme libre, séparée de son premier mari, avec deux élèves qu'elle avait formées, elle est peinte par son élève préférée des années plus tard, au milieu d'un cénacle presque exclusivement masculin. Le titre du tableau ajoute encore à ce qui ressemble à une sorte de déclassement posthume: "Madame Vincent, élève de son mari ». Cette parenthèse enchantée pour les artistes femmes que furent, selon certaines historiennes de l'art, les deux dernières décennies du XVIII siècle, était déjà bien refermée.

C'est au départ une question de terminologie qu'aborde Floriane Philippe dans son étude: "Pour une approche génétique de l'ébauche». Le sens du mot, à la fin du XVIII ${ }^{\mathrm{e}}$ siècle, semble fixé; l'ébauche constitue le commencement de l'œuvre et appartient alors à la même matière, tandis que l'esquisse est réalisée sur un support indépendant. Les deux sens vont cependant se brouiller au XIX siècle, l'un et l'autre des termes servant à exprimer le même moment trouble dans la préparation de l'œuvre : première forme, si l'on s'en tient à une acception matérielle ou bien première idée, si on la traite comme une opération de l'esprit. Très vite le champ couvert par le terme ébauche va s'étendre, désignant parfois également, dans la préparation d'une peinture, l'opération où, par-dessus le dessin, sont indiqués les couleurs ou les effets, recouvrant plus généralement tout ce qui relève de l'inachèvement. Esquisse et ébauche vont alors souvent être employés dans un sens métaphorique, y compris chez un esprit aussi subtil qu'Eugène Delacroix. D'une fonction, c'est-à-dire une étape dans le processus de création, l'ébauche aussi bien que l'esquisse vont finir par désigner une manière ou des effets. On voit alors tout le paradoxe qui entre dans ce nouveau sens que prend alors progressivement le terme: alors que par définition il désignait un moment transitoire, appelé à être perdu, du processus de création, l'ébauche va se rattacher à des œuvres au statut complexe, œuvres dont l'inachèvement peut relever ou bien d'un renoncement ou bien d'une décision d'arrêt en l'état. L'œuvre de Gustave Moreau, ici convoquée à travers plusieurs exemples, dont Apollon et les Muses (vers 1856, Paris, Musée Gustave Moreau), laisse apparaître, à côté du dieu les autres figures ainsi que le paysage laissés dans l'indétermination. Cela fait alors de cette œuvre, comme de bien d'autres chez Moreau, une sorte d'archive du laboratoire qu'est la création en même temps qu'une œuvre qui vient jouer non pas simplement sur l'esthétique de l'inachèvement mais aussi, comme par anticipation, sur celle de la suggestion. Cette 
étude montre l'apport qui peut être celui de la génétique de la création littéraire dans la compréhension des processus de création dans le champ des arts visuels. L'étude du champ lexical et de l'évolution du sens de termes appariés comme esquisse et ébauche, puis l'exemplification faite à partir d'un cas ne sont qu'une illustration, aussi riche d'attendus théoriques que vivifiante par l'enrichissement du regard qu'elle entraîne, de l'utilité de cette hybridation des méthodes issues des études littéraires et de questions propres à l'histoire de l'art.

6 Autre exemple d'hybridation: l'histoire monétaire et l'histoire des médailles, ou comment l'introduction en 1895-1898 d'une monnaie d'or engage des problèmes formels et iconographiques, mélangés à des attendus politiques qui se manifestent à la fois dans la commande de cette nouvelle pièce et dans la réception qui lui est faite. Dans son étude, «La fortune critique d'une monnaie d'or: le coq gaulois de Jules-Clément Chaplain à l'épreuve de la presse (1895-1914) ", Katia Schaal se penche sur la décision faite par le gouvernement républicain, sous le ministère de Léon Bourgeois, de passer commande à trois artistes différents pour trois pièces de monnaie de métal différentes, ornées d'un symbole républicain. La monnaie d'or, qui est ici l'objet de l'étude, revint au sculpteur Chaplain, un ancien élève des Beaux-Arts, ancien prix de Rome qui s'était fait remarquer pour ses médailles aux expositions universelles de 1867 et 1878 . Préparée pendant l'hiver 1895 par le ministre des finances, Paul Doumer, soucieux de concevoir au mieux les symboles et les emblèmes des nouvelles pièces, conscient de l'extraordinaire vecteur de diffusion d'un message politique qu'elles représentaient, pesa directement sur le choix des deux figures, au droit un profil de la République et au revers un coq gaulois. Le type d'emblème choisi, restait à en préciser l'image, ce que fit Chaplain, dans diverses esquisses qui lui permirent d'aboutir à la conception finale, laquelle devait naturellement se plier aux contraintes de la frappe. Toutes sortes d'enjeux, mis en lumière par K. Schaal, entourent alors la réception de la médaille : si sa genèse est indissociable des doctrines de l'art social dont Roger-Marx est une des voix les plus influentes, sa conception et sa réception se font dans le soupçon des académiciens de voir sacrifier la dimension artistique, une monnaie devant toujours être également une médaille. Et naturellement, le choix des emblèmes ne pouvait que provoquer des réactions aussi bien chez les adversaires de la République que chez les défenseurs de la laïcité, hostiles à ce que la devise « Dieu protège la France » vint orner la tranche des pièces de vingt francs. Les étapes de la fabrication de masse, avec les problèmes industriels qu'elle ne manqua pas de poser et la réception par l'usage avant le retrait des pièces d'or en 1914, complètent l'étude de la "carrière » de cette piècemédaille. Cette étude, s'il faut la résumer en un trait, montre comment un artiste chercha à concilier les valeurs d'usage et symbolique avec la valeur artistique ; ce que l'histoire de l'art peut gagner en intégrant des problématiques qui longtemps furent le domaine propre des historiens. Quand l'étude des formes et des techniques croise celle de l'allégorie politique ou encore de la presse au XIXe siècle.

7 L'étude d'Hélène Fernandez apporte une contribution de premier plan à la connaissance d'un des grands peintres et illustrateurs français de la fin du XIX siècle et du début du $\mathrm{XX}^{\mathrm{e}}$ siècle - «Une descente dans la vérité : contribution à l'étude de l'œuvre de Jean-Louis Forain à partir de la collection de dessins du musée d'Orsay (conservés au département des arts graphiques du musée du Louvre) ». Le travail de catalogage, indispensable, imposait de rapprocher les dessins des planches parues dans les différents journaux avec lesquels l'artiste collaborait (Le Courrier français, Le Fifre, L'Écho de Paris, Le Figaro, L'Enquête sur la monarchie...). Enfin, le surplus de dessin est relié 
avec les planches imprimées, les feuilles étant classées selon un ordre thématique : les scènes de la vie contemporaine, la justice, les portraits, les sujets religieux inspirés du Nouveau Testament, le nu féminin. Ces deux corpus, on le voit, se rejoignent alors très souvent dans ces thèmes, notamment les scènes de la vie contemporaine, traitées dans un esprit naturaliste. Dans Au Café de la Nouvelle Athènes (cat. 61) une feuille sur laquelle Forain a campé la société artistique et littéraire qui s'y pressait, on devine à la fois l'univers des mœurs dont le lieu, avec d'autres, était le théâtre, fait d'adultères ou d'histoires monnayées, et la bohème artistique dont elle était un foyer emblématique. Avec Degas, autre habitué du Café de la place Pigalle, Forain représente des hommes qui fréquentent de jeunes danseuses (cat. 8, 10-11, 27 et 51). Là encore dans un esprit naturaliste, il décrit les existences tristes des demi-mondaines, comme cette «Jeune fille faisant la vaisselle le matin, et la même, en danseuse, le soir" (cat. 30), qui illustrerait un poème de Degas et qui sera repris dans Le Fifre en 1889. Des couples clandestins se retrouvent également en couverture dans L'Écho de Paris (cat. 35) ou dans Le Rire (cat. 48), plus encore dans les séries «L'amour à Paris », « Mœurs conjugales » ou «Les joies de l'adultère » (Le Courrier français). Les artistes se substituent parfois aux messieurs à redingotes dans la recherche des amours faciles, comme cet « Homme près d'une femme devant un tableau sur un chevalet» (cat.6), donné en 1888 au même Courrier français. Au peintre qui lui fait vraisemblablement des avances, le jeune modèle résiste en lui rappelant les risques judiciaires qu'il prend ; et l'homme chasse le modèle, refusant de de payer une amende élevée là où un peu de prison ou la perte des droits civiques ne semble guère l'embarrasser, invitant pour finir la jeune femme à aller « poser » chez Bouguereau. Nous sommes bien ici chez Les Goncourt, dans l'univers de Manette Salomon.

8 L'équipe de recherche de l'École du Louvre a été constituée il y a dix ans. Elle venait prolonger et enrichir les thèses d'établissement qui y avaient été menées depuis sa fondation. De nouvelles thèses y sont soutenues depuis, le plus souvent sous la forme de co-encadrements universitaires. Des programmes de recherche y sont menés, des colloques et des séminaires y prennent place chaque année. Les Cahiers de l'École du Louvre, avec les travaux soutenus qui font l'objet d'une publication, viennent achever et rendre plus visible encore toute l'activité de cette communauté. S'il faut chercher une philosophie, tout au moins un principe qui entoure ces différentes activités de recherche, il est selon nous dans le dialogue que les historiens de l'art doivent en permanence entretenir, avec les autres disciplines sans doute, mais déjà au sein même de la discipline, avec les différentes spécialités, dans une grande variété de champs et de domaines. Ce principe est ce que cherche à favoriser l'école. Les antiquisants y discutent avec les contemporanéistes; les spécialistes d'art africain avec les américanistes; les chercheurs menant des thèses sur les arts décoratifs avec leurs confrères relevant des études cinématographiques; les historiographes avec les spécialistes de la matérialité des œuvres... Au moment où l'École du Louvre s'apprête à inaugurer, à l'automne 2021, de nouveaux locaux pour son centre de recherche et sa bibliothèque, dont l'ouverture sera l'occasion des Journées de la recherche tenues au mois de novembre, ce numéro de varia vient non seulement témoigner de la richesse de la recherche développée par les doctorants, docteurs et membres de notre équipe, mais surtout rappeler la nécessité des passerelles inattendues, de la curiosité pour les domaines éloignés, de la perturbation qui vient des méthodes éprouvées ailleurs, des hybridations qui en résultent et des échanges qui donnent au travail scientifique sa véritable plénitude. 


\section{AUTEUR}

\section{FRANÇOIS-RENÉ MARTIN}

Coordinateur de la recherche à l'École du Louvre 